\title{
Evolution on a Smooth Landscape
}

\author{
David A. Kessler \\ Minerva Center for the Study of Mesoscopics, Fractals, and Neural Networks and Dept. of Physics, Bar-Ilan Univ., Ramat \\ Gan, Israel \\ Herbert Levine, Douglas Ridgway and Lev Tsimring \\ Institute for Nonlinear Science, University of California, San Diego La Jolla, CA 92093-0402
}

\begin{abstract}
We study in detail a recently proposed simple discrete model for evolution on smooth landscapes. An asymptotic solution of this model for long times is constructed. We find that the dynamics of the population are governed by correlation functions that although being formally down by powers of $N$ (the population size) nonetheless control the evolution process after a very short transient. The long-time behavior can be found analytically since only one of these higher-order correlators (the two-point function) is relevant. We compare and contrast the exact findings derived herein with a previously proposed phenomenological treatment employing mean field theory supplemented with a cutoff at small population density. Finally, we relate our results to the recently studied case of mutation on a totally flat landscape.
\end{abstract}

Key Words: Evolution, Birth/Death Processes, Mean-Field, Population Dynamics

\section{INTRODUCTION}

In a recent paper, [1] we introduced a model to describe evolution on a smooth landscape. This model was motivated by the results of recent experiments [2] on the evolution of fitness in populations of RNA viruses. In these experiments, the population shows secular changes in birth rate, which is identified by the biologists as the logarithm of fitness. These experiments showed a rapid initial increase of birth rate, followed by a sharp transition to a regime of significantly slower, approximately linear, increase. The initial rapid rise in birth rate was interpreted by us to be a result of the exponentially quick dominance of the population by the most "fit" (most rapidly reproducing) members of the initial population. These most "fit" individuals produce many more offspring, so they quickly dominate, in accord with a simple picture of the working of selection in a population. The subsequent slow rise was interpreted as being due to the effect of mutations which, as the mutation rate is low, act on a slower time scale.

The relative smoothness of the increase of birth rate in this second, linear, regime indicates that the "fitness" landscape must be fairly smooth. It is clear from many studies of evolution [3 5 ] in rough, "glassy" landscapes, that increases in fitness in such cases are expected to be sudden, with long intervals of little or no improvement in between. While it is clear that the population should smoothly "climb the hill" in a smooth landscape, no attention seems to have been focused on the precise dynamics of such hill-climbing. In particular, what is the speed with which the population climbs, and on what does it depend?

To begin to address these questions, we formulated [1] a very simple discrete model of evolution in a smooth landscape. This model exhibited both the initial rapid "collapse" of the population onto its most fit members, and the subsequent smooth rise in birth rate which characterized the RNA virus experiments (see Fig 1.). In addition, we constructed a continuum mean-field treatment which, when suitably doctored with a cutoff, appeared to give a qualitatively correct description of the dynamics. As we were concerned with the gross qualitative features of the model and its possible relevance to the experiment, we did not investigate the precise extent of the apparent agreement nor indicate in any detail as to where the cutoff came from.

In this paper, we return to the analysis of our discrete model. Our analysis provides an exact description of the dynamics at asymptotically long times. This asymptotic dynamics indicates that the population does indeed increase its birth rate in a linear fashion, and provides a prediction of the rate of such increase. It also provides a description of the stochastic departures from this linear behavior. The most striking result of this analysis is that the rate of increase of birth rate (the velocity of climbing of the fitness hill) depends crucially on the population size (increasing essentially linearly with the population). Naive mean-field theory is however completely independent of $N$, the population size. Indeed, we show explicitly how naive mean-field theory gives rise to a finite-time divergence (or in another variant of the model, an exponential blowup) of the fitness and how the true dynamics cures this unphysical behavior through terms which are formally lower order in $1 / N$. These terms become relevant on relatively short time-scales and drive 
the system to an $N$ dependent asymptotic state. It is important to note that even the cutoff version of mean-field theory, which does not suffer from the blowup phenomenon, does not correctly predict this asymptotic state, due to the neglect of a specific two-body correlation term which dominates at long-time. This diagnosis of the failings of mean-field theory is the second major product of our analysis.

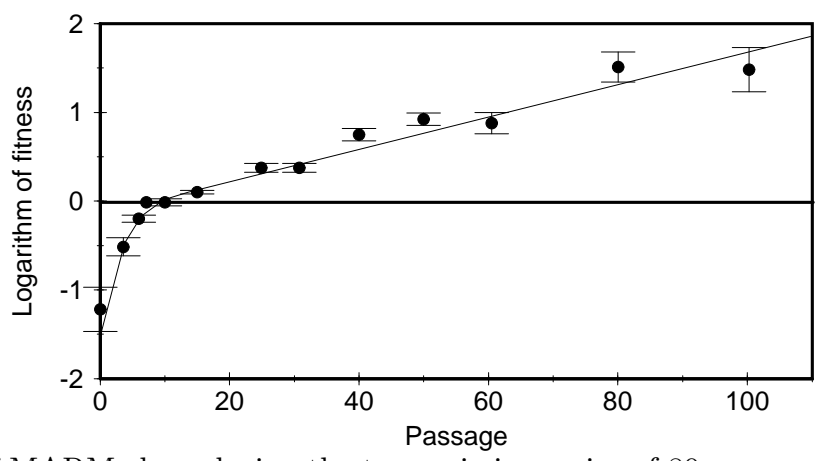

FIG. 1. Evolution of fitness of MARM clone during the transmission series of 80 passages on HeLA cells (Fig. 2b of 2 with permission from PNAS)

\section{PRELIMINARIES}

We begin our exposition by presenting the discrete model of evolution which forms the basis of our discussions. We also outline the naive mean-field theory and the modified cutoff version we analyzed in our previous work [1].

\section{A. The Discrete Model}

The discrete model whose behavior we will primarily focus on is a slight variant of that discussed in our earlier work. The original model, as well as another variant, the flat landscape model, can be easily analyzed in a manner parallel to that with which we shall treat the version we present here. The discussion of these other variants we will thus postpone to a later section.

Our model consists of a fixed population of $\mathrm{N}$ individuals. Although in the experiments the population varied between two essentially fixed values, the fixed population constraint is obviously the simpler case to study first, and seems to preserve the qualitative features of the more general case. The individuals are characterized by their birth rates, which the experimentalists associate with the logarithm of the "fitness" of the individual. Each individual gives birth at his characteristic rate in a standard Poisson process. The baby, due to mutation, may have a birth rate which is $\pm \Delta$ that of his parent. The overall mutation probability for a baby is a constant $\mu$. By rescaling time by $\Delta$, we may take the change in the baby's birth rate to be \pm 1 , so that the birth rates are restricted to be integers. We do not allow a baby to mutate to 0 birth rate, so in fact the birth rates are all natural numbers. As we work at fixed population size $N$, each birth is occasioned by the unfortunate demise of some member of the group. The victim is chosen at random from among all members of the population (including the new-born baby) independent of their fitness rating.

To get some idea of the dynamics of this model, we show in Figure 2 the results of a simulation of this model with a population size of $N=50$. Starting from a population of identical individuals, the average mean-birth-rate (note: average means over different realizations, mean denotes the mean for all individuals in a given simulation) accelerates quickly, followed by a gradual deacceleration to a regime of constant velocity, i.e. a linear increase of mean-birth-rate with time. Looking also at the variability of the population, we calculate the average sample width-squared, which increases rapidly from zero and then saturates at some finite value. We will see later that the transient phase is complicated, but the asymptotic state is always one of constant velocity.

\section{B. Mean-Field Theory}

It is easy to construct a mean-field theory for the simple process outlined above. Let $P(n, t)$ be the number of individuals with birthrate $n$ at time $t$. Then, in the absence of mutations, it is clear that 


$$
\dot{P}=(n-\bar{n}) P(n)
$$

where $\bar{n}$ is the instantaneous mean value of $n$. The presence of mutations means that a fraction $\mu$ of the $n P(n)$ newborns will leave birth-rate $n$, half moving up to $n+1$ and half down to $n-1$. Thus,

$$
\dot{P}(n)=(n-\bar{n}) P(n)-n \mu P(n)+\frac{1}{2} \mu[(n+1) P(n+1)-(n-1) P(n-1)]
$$

or, in continuum language, replacing the discrete $n$ by $x$

$$
\dot{P}(x)=(x-\bar{x}) P(x)+\frac{1}{2} \mu(x P(x))^{\prime \prime}
$$

This equation is essentially identical to that of the mean-field theory for Diffusion-Limited Aggregation (DLA) [6] in the far-field (large- $x$ ) regime. It is not surprising, therefore, that both the mean-field DLA [7] and our mean-field Equation (2) share the property that an initial pulse accelerates to infinite velocity in finite time. We will return to our mean-field Equation (2), and in particular demonstrate explicitly the finite-time singularity, in Section VI.

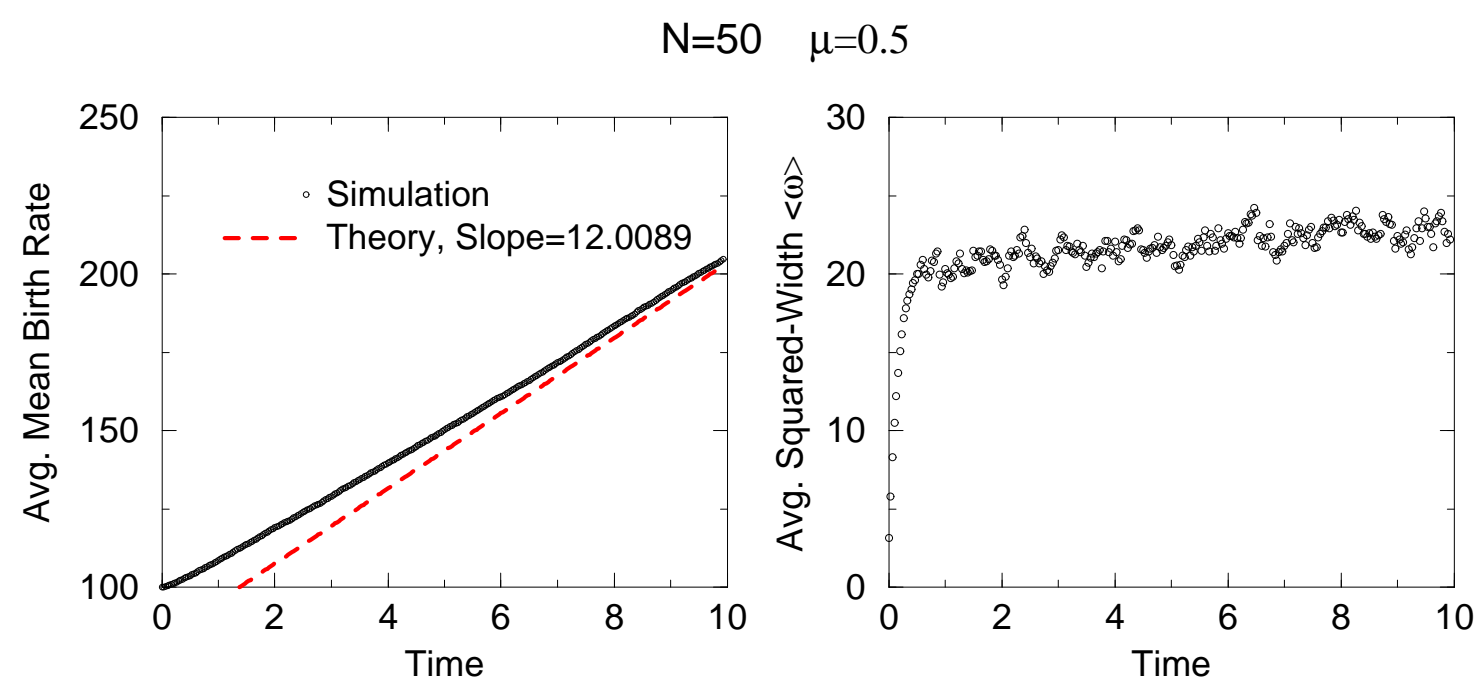

FIG. 2. a) Mean birth-rate for population of $N=50$ individuals as a function of time, with mutation rate $\mu=.5$, averaged over 200 realizations. Dotted line shows theoretical predicted rate of increase of mean-birth-rate.

b) Width-squared from same simulation.

For the moment, it is important to inquire as to the origin of this unphysical behavior. From a mathematical standpoint, the essential problem is the term $x P$, which is unbounded. Physically, although it is extremely unlikely for an individual to find itself far in front of the rest of the population, should it happen, then that individual's growth rate would be enormously greater than anyone else's. Thus, this very unlikely scenario makes a significant contribution to the ensemble average $P(x)$ since its contribution is the product of the (tiny) probability of this configuration times the (huge) number of individuals at this site. In the model, however, the contribution of such an outlyer to the ensemble average remains negligible since the total number of individuals in any given configuration is limited to $N$. From this discussion, however, we can surmise that the velocity of propagation should be a strongly increasing function of $N$. Since mean-field theory is a theory for infinite $N$, mean-field theory is in some sense giving the correct answer, which is an infinite velocity at infinite $N$. While perhaps correct in this sense, such an answer is of course of little use.

There are various prescriptions one might employ to remove this ugly feature of the mean-field theory, some of which have been suggested in the context of mean-field DLA [7, 8]. In our earlier work on a variant of this model [1], we employed the stratagem of cutting off the $(x-\bar{x}) P$ term when $P(x)$ was under some threshold value $P_{*}$. It is clear from the outset, however, that for this system such a resulting theory cannot give rise to a steadily-propagating solution, in contradiction with simulation. Furthermore, it is not clear how to justify this prescription (or any of the others that have been suggested) on a first-principles basis. Thus, we leave mean-field theory for the interim, and 
study in detail some simpler, though nontrivial limits of the model, those of a very small number of individuals and the case of very small mutation rate $\mu$.

\section{SIMPLE LIMITING CASES}

In this section, we discuss the simplest possible cases for the model as described above, that of populations of either one or two individuals or alternatively the case of extremely small mutation rate. As we shall see, this exercise allows us to determine exactly the nature of the long-time state and is furthermore quite useful in pointing out what one needs to do to make sense of the utter failure of simple mean field ideas.

\section{A. The Case of $N=1$}

As an introduction to the case of 2 individuals, let us quickly see what happens for one individual. Although trivial to work out, it is actually a useful signpost on the road to the more interesting case of $N=2$.

For $N=1$, we can write down the exact master equation for the Markov process. As there is only one individual, a configuration is labeled by the position of that one particle, so we can express everything in terms of $f(n, t)$, the probability that the individual is at site $n$ at time $t$. As the only way the individual can move to a new spot is by giving birth, which occurs at a rate $n$ and then dying in favor of his child ( a $50 \%$ probability) so that his child can survive and, with probability $\mu$, step to the left or right. Thus, the exact master equation reads

$$
\dot{f}(n)=-\frac{1}{2} \mu n f(n)+\frac{1}{4} \mu[(n+1) f(n+1)+(n-1) f(n-1)]
$$

or, in continuum terms,

$$
\dot{f}(x)=\frac{1}{4} \mu(x f(x))^{\prime \prime}=\frac{1}{4} \mu\left(2 f^{\prime}+x f^{\prime \prime}\right)
$$

This is very reminiscent of the mean-field equation, Eq. 3 except for the extra factor of $1 / 2$ in the diffusion constant, but, more importantly, the total absence of the troublesome $x P$ term! The reason is clear: with only one individual, there can be nobody out in front of the pack out-reproducing everyone. In fact, the birth process itself is completely irrelevant in changing the configuration, as one of the two individuals existing post-birth is immediately tagged for death, leaving the configuration unchanged.

As we shall soon see, this absence of the $x P$ term is a very general result, and, in fact, the equation of motion for long times is, in the general case, exactly the same as for the $N=1$ case, up to changes in the values of the coefficients.

\section{B. $\mathbf{N}=2$ : The Small $\mu$ Limit}

The case of $N=2$ is less trivial. A general configuration is specified by the positions of both individuals, so that it constitutes a 2-dimensional field. However, things simplify tremendously in the small $\mu$ limit. The point is that if $\mu$ were exactly 0 , then the population would quickly collapse, with both individuals finding themselves at the same location (most likely that of the fitter of the two). For small $\mu$, every so often one of the two begins to wander away, but it can't get far before the population collapses again. So, for small $\mu$, we need consider only 2 types of configurations: 1)the two individuals on the same site, and 2) the individuals on nearest-neighbor sites. Call the probability the individuals are on the same site $x, f(x)$. The probability that they are on $x$ and $x+1$ we call $g(x)$. The master eqn. for the process is then:

$$
\begin{aligned}
& \dot{f}(x)=-\frac{4}{3} \mu x f(x)+\frac{1}{3}(1-\mu)(x g(x-1)+x g(x))+\frac{\mu}{6}((x+1) g(x)+(x-1) g(x-1)) \\
& \dot{g}(x)=\frac{2}{3} \mu(x f(x)+(x+1) f(x+1))-\frac{1}{3}(1-\mu)((x+1)+x) g(x)-\frac{\mu}{6}((x+1)+x) g(x)
\end{aligned}
$$

Notice we have forbidden the transitions from " $g$ " to the state with a space between the 2 individuals, to conserve probability. We can of course combine terms above, but it is written so that each term in the $f$ equation corresponds 
directly to the same term in the $g$ eqn. As we have argued, $g$ is small, of order $\mu$, so since we are working only to linear order in $\mu$, we shall drop all terms involving $\mu g$. Then the above simplifies nicely to

$$
\begin{aligned}
& \dot{f}(x)=-\frac{4}{3} \mu x f(x)+\frac{1}{3}(x g(x-1)+x g(x)) \\
& \dot{g}(x)=\frac{2}{3} \mu(x f(x)+(x+1) f(x+1))-\frac{1}{3}((x+1) g(x)+x g(x))
\end{aligned}
$$

Notice now that there is an exact time-independent and $x$-independent solution to these eqns., namely $g=2 \mu f=$ const. Of course, this solution is not normalizable, but as we will see, this is what the system is approaching for long times. This motivates us to guess that

$$
g(x) \approx 2 \mu f(x)+\mu f^{\prime}(x)\left(A_{1}+\frac{A_{2}}{x}\right)+\mu f^{\prime \prime}(x) B
$$

or, equivalently (to the order we will need, (see below))

$$
2 \mu f(x) \approx g(x)+g^{\prime}(x)\left(C_{1}+\frac{C_{2}}{x}\right)+g^{\prime \prime}(x) D
$$

Notice that this is an expansion in derivatives, which are assumed to decrease in magnitude with increasing order. The justification for this is the statement above, that $f$ and $g$ are approaching constants at long time, so the expansion is essentially one in inverse powers of $t$. We will see this explicitly later. It is also a large $x$ expansion. In order to compute the velocity, we will need to keep terms up to $x^{-1}$ in $f^{\prime}, g^{\prime}$ and up to $x^{0}$ in $f^{\prime \prime}, g^{\prime \prime}$. We can relate the coefficients $A$ and $B$ to $C$ and $D$ by substituting the first expression in the second and expanding. We find $A_{1}=-2 C_{1}$, $A_{2}=-2 C_{2}$, and $B=-2 C_{1}^{2}-2 D$.

The idea now is to substitute the above relation in the equation of motion for $f$ and $g$. We note that $\dot{f}$ is proportional to $\mu$, but $\dot{g}$ has terms zeroth order in $\mu$. For this to be consistent with Eq. (\$) relating $g$ to $f$, we must make these zeroth order terms vanish. This gives us equations for the coefficients $C$ and $D$. In more detail,

$$
\begin{aligned}
\dot{g}(x) & =\frac{1}{3}\left[(2 x+1) g^{\prime}\left(C_{1}+\frac{C_{2}}{x}\right)+g^{\prime \prime} D+(x+1)\left(g^{\prime}+C_{1} g^{\prime \prime}\right)+\frac{x}{2} g^{\prime \prime}\right] \\
& =\frac{1}{3}\left[x g^{\prime}\left(2 C_{1}+1\right)+g^{\prime}\left(C_{1}+2 C_{2}+1\right)+x g^{\prime \prime}\left(2 D+C_{1}+\frac{1}{2}\right)\right]
\end{aligned}
$$

Thus, $C_{1}=-\frac{1}{2}, C_{2}=-\frac{1}{4}$, and $D=0$, so that $A_{1}=1, A_{2}=\frac{1}{2}$, and $B=\frac{1}{2}$. Substituting the relation for $g$ in term of $f$ in the eqn. for $\dot{f}$ yields

$$
\begin{aligned}
\dot{f}(x) & =\frac{2 \mu x}{3}\left[f^{\prime}\left(A_{1}+\frac{A_{2}}{x}\right)+f^{\prime \prime} B\right]-\frac{\mu x}{3}\left[2 f^{\prime}+A_{1} f^{\prime \prime}\right]+\frac{\mu x}{6} 2 f^{\prime \prime} \\
& =\frac{\mu x}{3}\left[f^{\prime}\left(2 A_{1}+\frac{2 A_{2}}{x}-2\right)+f^{\prime \prime}\left(2 B-A_{1}+1\right)\right] \\
& =\frac{\mu}{3}\left[f^{\prime}+x f^{\prime \prime}\right]
\end{aligned}
$$

The last eqn. directly implies that the velocity $v$ is $\mu / 3$, since

$$
v=\frac{d}{d t} \int d x x f(x)=\frac{\mu}{3} \int x f^{\prime}+x^{2} f^{\prime \prime}=\frac{\mu}{3}
$$

where the last result comes from integration by parts and the fact that $f$ is normalized to unity at lowest order. Furthermore, there exists a similarity solution for this $f$ equation, (about which we will discuss more later) with $x$ scaling like $t$, so that a long-time expansion is also a large- $x$ expansion as advertised. Also, as advertised, the form of the equation of motion for $f$ is exactly as in the $N=1$ case. What we have therefore, is essentially a bound-state of the two individuals propagating together at constant velocity (on average) with the center of mass performing (inhomogeneous) diffusion. The squared-width of this bound-state, the expectation value of the squared-distance between the two individuals, is $g /(f+g)$, which to the order we are calculating is simply $2 \mu$. 


\section{General $N$ : The Small $\mu$ Limit}

We can extend the calculation in the previous section to arbitrary $N$. To lowest order in $\mu$, we have to consider only those states where the individuals are distributed between 2 nearest-neighbor sites. We label the probability of being in the state with $N-k$ individuals at $x$ and $k$ individuals at $x \pm 1$ by $g_{k}^{ \pm}(x),(0<k<N / 2)$. The probability of the state with all $N$ individuals at $x$ is $f(x)$. If $N$ is even, we also have the symmetric state $g_{N / 2}(x)$, but for our purposes it is enough to do the odd $N$ case. Again, we can write down the master equation for the process, ignoring transitions out to other states, which are down by additional powers of $\mu$. The equations of motion then read

$$
\begin{aligned}
\dot{f}(x)=\frac{1}{N+1}[ & \left.-N^{2} \mu x f(x)+(N-1) x\left(g_{1}^{+}(x)+g_{1}^{-}(x)\right)\right] \\
\dot{g}_{1}^{ \pm}(x)=\frac{1}{N+1}[ & \left.\frac{1}{2} N^{2} \mu x f(x)-(N-1)(2 x \pm 1) g_{1}^{ \pm}(x)+2(N-2) x g_{2}^{ \pm}(x)\right] \\
\dot{g}_{k}^{ \pm}(x)=\frac{1}{N+1}[ & -(N-k) k(2 x \pm 1) g_{k}^{ \pm}(x)+(N-k-1)(n+1) x g_{k+1}^{ \pm}(x) \\
& \left.+(N-k+1)(n-1)(x \pm 1) g_{k-1}^{ \pm}(x)\right] \\
\dot{g}_{\frac{N-1}{2}}^{ \pm}(x)=\frac{1}{N+1}[ & -\frac{N-1}{2} \cdot \frac{N+1}{2}(2 x \pm 1) g_{\frac{N-1}{2}}^{ \pm}(x)+\frac{N-1}{2} \cdot \frac{N+1}{2} x g_{\frac{N-1}{2}}^{\mp}(x \pm 1) \\
& \left.+\frac{N-3}{2} \cdot \frac{N+3}{2}(x \pm 1) g_{\frac{N-3}{2}}^{ \pm}(x)\right]
\end{aligned}
$$

where the middle equation holds for $k=2, \ldots,(N-3) / 2$. All the $g$ 's are again slaved to $f$, so we write

$$
g_{k}^{ \pm}(x)=\mu f(x)\left(A_{k}^{0} \pm \frac{A_{k}^{1}}{x}+\frac{A_{k}^{2}}{x^{2}}\right)+\mu f^{\prime}(x)\left( \pm B_{k}^{0}+\frac{B_{k}^{1}}{x}\right)+\mu f^{\prime \prime}(x) C_{k}
$$

We have used the reflection symmetry to determine the \pm signs in this equation. Essentially, they came from the fact that the \pm 1 's in the equations of motion are down by $1 / x$ from the leading terms. The procedure is exactly the same as before. The coefficients $A_{k}^{i}, B_{k}^{i}$, and $C_{k}$ are determined by the requirement that the $\dot{g}_{k}^{ \pm}$must all vanish to leading order. We find, after a mess of algebra, that

$$
\begin{aligned}
A_{k}^{0} & =\frac{N^{2}}{2 k(N-k)} \\
A_{k}^{1} & =-\frac{N(N-2 k)}{2 k(N-k)} \\
A_{k}^{2} & =-\frac{N(k-1)}{2 k} \\
B_{k}^{0} & =\frac{N}{2(N-k)} \\
B_{k}^{1} & =-\frac{N(N-k-2)}{4(N-k)} \\
C_{k} & =\frac{N-k-1}{N-k}
\end{aligned}
$$

We see from this that the profile is asymmetric, with the left (trailing) side larger. However this asymmetry is proportional to $1 / x$ and so slowly decays in time.

Now we plug all this into the equation of motion for $f$, finding that

$$
\dot{f}=-v f^{\prime}+D(x f)^{\prime \prime}
$$

where the velocity, $v$, is given by

$$
v=\mu \frac{N(N-1)}{2(N+1)}
$$


and so increases (roughly) linearly with $N$, in accord with our general expectations above. The diffusion constant of the center of mass, $D=\frac{N}{2(N+1)}$ is roughly constant with $N$. The (unnormalized) long-time similarity solution for $f$ is

$$
f(x, t)=\frac{1}{x}\left(\frac{x}{t}\right)^{v / D} e^{-x / D t}
$$

We see that the spreading of the center of mass is linear in time.

Unfortunately, we see that the $g$ 's are actually of order $O(\mu N)$, so the expansion is not uniformly valid in $N$. Nevertheless, it is clear that the form of the equation of motion is true to all orders in $\mu$, with just the coefficients $v$ and $D$ varying. The task therefore is to compute these coefficients for arbitrary $\mu$. For the case $N=2$, we can generalize our analysis above to accomplish this, so we return to that case.

\section{Two Individuals, Arbitrary $\mu$}

For the $N=2$ case, we can straightforwardly generalize our small $\mu$ solution given above to the case of arbitrary $\mu$. The relevant variables are $f_{n}(x, t),(n=0,1, \ldots)$, the probability of an individual at $x$ and one at $x+n$. The equations of motion are:

$$
\begin{gathered}
\dot{f}_{0}(x)=\frac{1}{3}\left[-4 \mu x f_{0}(x)+x(1-\mu) \sum_{n=1}^{\infty}\left[f_{n}(x)+f_{n}(x-n)\right]+\frac{1}{2} \mu\left((x+1) f_{1}(x+1)+(x-1) f_{1}(x-1)\right)\right] \\
\dot{f}_{1}(x)=\frac{1}{3}\left[2 \mu\left(x f_{0}(x)+(x+1) f_{0}(x+1)\right)-(1+\mu)(2 x+1) f_{1}(x)\right. \\
\quad+\frac{1}{2} \mu \sum_{n=1}^{\infty}\left[x f_{n}(x)+(x+1) f_{n}(x+1)+x f_{n}(x-n)+(x+1) f_{n}(x-n+1)\right] \\
\left.\quad+\frac{1}{2} \mu\left((x+2) f_{2}(x)+(x-1) f_{2}(x-1)\right)\right] \\
\dot{f}_{n}(x)=\frac{1}{3}\left[-(1+\mu)(2 x+n) f_{n}(x)+\frac{1}{2} \mu\left((x+n+1) f_{n+1}(x)+(x-1) f_{n+1}(x-1)\right)\right. \\
\left.+\frac{1}{2} \mu\left((x+n-1) f_{n-1}(x)+(x+1) f_{n-1}(x-1)\right)\right]
\end{gathered}
$$

where in the last equation, $n \geq 2$. Again, there is an exact steady-state solution, with all the $f_{n}$ independent of $x$. If we choose the ansatz $f_{n}=A r^{n} f_{0}$, the equation for $\dot{f}_{n}$ reads

$$
0=-\frac{1}{6}(2 x+n) A f_{0} r^{n-1}\left(2(1+\mu) r+\mu r^{2}+\mu\right)
$$

which has the solution, (defining $\chi \equiv 1 / \mu$ )

$$
r=\chi+1-\sqrt{\chi^{2}+2 \chi}
$$

For small $\mu, r \approx \mu / 2 \ll 1$, and $r$ increases monotonically with $\mu$. This is in accord with our expectation for a rapid falloff of $f_{n}$ with $n$ for small $\mu$. For the maximum physical $\mu=1, r=2-\sqrt{3} \approx 0.27$. Plugging this form for $f_{n}$ into either of the two remaining equations yields $A=4(1-r) /(1-3 r)$. The normalization condition that $\sum_{n} f_{n}=1$ then determines $f_{0}=(1-3 r) /(1+r)$. Note that $f_{0}$ and $A$ are positive, as they should be, for all physical values of $\mu$. We can then calculate the exact squared-width of the bound state,

$$
<n^{2}>=\sum_{n} n^{2} f_{n}=2 \mu .
$$

Note that the first-order result derived above in the small $\mu$ limit is in fact exact to all orders!

We can again generalize to the long-time limit where the derivatives of $f$ are small, but non-zero. We write

$$
f_{n}=r^{n}\left(A f_{0}+B_{n} f_{0}^{\prime}+C_{n} f_{0}^{\prime} / x+D_{n} f_{0}^{\prime \prime}\right)
$$


If we assume the expected form for $\dot{f}_{0}$ :

$$
\dot{f}_{0}=-v f_{0}^{\prime}+D\left(x f_{0}\right)^{\prime \prime}
$$

we can obtain a set of equations for the coefficients $B_{n}, C_{n}$ and $D_{n}$ along with $\tilde{v}$ and $D$ by matching the coefficients of $f_{0}$ and its derivatives. After much algebra, we find

$$
\begin{aligned}
B_{n} & =n A / 2 \\
C_{n} & =\left(-\frac{1}{8} n^{2}+\frac{(3 \chi-1) r}{4\left(1-r^{2}\right)} n+\frac{r^{2}(2 \chi-3)}{2(1-r)^{2}(1+r)(1-3 r)}\right) A \\
D_{n} & =\left(\frac{1}{8} n^{2}+\frac{(\chi-3) r}{4\left(1-r^{2}\right)} n+\frac{r^{2}(2 \chi-1)}{2(1-r)^{2}(1+r)(1-3 r)}\right) A \\
v & =\mu / 3 \\
D & =\mu / 3
\end{aligned}
$$

Just as was the case with the width, the coefficient of the drift $\tilde{v}$ and diffusion $D$ are given exactly by the linear in $\mu$ expressions found above! Such simple results cry out for a simpler derivation, which we now present.

\section{MOMENT EQUATIONS}

As we have seen, the dynamics of our discrete evolution model gives rise to a pulse of a typical width; the center of this pulse propagates at long times with some velocity and also diffuses. Unfortunately, the methodology used to obtain these results (essentially studying the full master equation) cannot be used to obtain the macroscopic parameters of the velocity and diffusion constant in general, and also do not indicate anything about the pre-asymptotic state. What we will see now is that one can re-derive the same picture for the long-time limit of the model at arbitrary parameters. This derivation proceeds by working with moment equations for the probabilistic process. These moment equations are valid at all times, and so also shed light on the short-time dynamics of the system.

\section{A. The Width of the Pulse}

Staying for the moment with the $N=2$ case, let us focus on the average squared-width, $<n^{2}>$. We wish to calculate $\left\langle\dot{n}^{2}>\right.$, the rate of change of the squared-width with time. Given the definition of $<n^{2}>$ in terms of the $f_{n}$ 's, (see Equation (22)) we can obtain what we want from the equations of motion of the $f_{n}$ 's. We find

$$
\begin{aligned}
<\dot{n^{2}>} & =-\frac{2}{3} \sum_{n} \bar{x} n^{2} f_{n}(x)+\frac{4}{3} \mu \sum_{n} \bar{x} f_{n}(x) \\
& =-\frac{2}{3}<\bar{x} n^{2}>+\frac{4}{3} \mu<\bar{x}>
\end{aligned}
$$

where $\bar{x} \equiv x+n / 2$ is the average position of the two individuals making up the configuration $f_{n}(x)$. If we may factorize the expectation value $<\bar{x} n^{2}>\approx<\bar{x}><n^{2}>$, (a step we can justify for long times from our explicit solution above) we get

$$
<\dot{n^{2}}>=-\frac{2}{3}<\bar{x}><n^{2}>+\frac{4}{3} \mu<\bar{x}>
$$

which immediately yields the steady-state value of $\omega=2 \mu$, which we obtained in the previous section.

This argument can be easily extended to arbitrary N. We now define the squared-width, $\omega$, as the mean squared distance between pairs of individuals, taken over all $N(N-1) / 2$ pairs.

$$
\omega \equiv \frac{2}{N(N-1)} \sum_{i>j}\left(x_{i}-x_{j}\right)^{2}
$$

and so its ensemble average is 


$$
<\omega>=\frac{2}{N(N-1)} \sum_{C} P_{C} \sum_{i>j}\left(x_{i}-x_{j}\right)^{2}
$$

where the first sum is over all configurations $C$, weighted by their probability $P_{C}$. We are thus considering a 2-point function, instead of the full $\mathrm{N}$-individual joint probability distribution. We can calculate $\dot{\omega}$ by considering the effect of each possible event on $\omega$. We find, summing over all events where $i$ is born and $j$ dies,

$$
\begin{aligned}
<\dot{\omega}>=\frac{2}{N(N-1)(N+1)} \sum_{C} P_{C} \sum_{i, j} x_{i}[ & (1-\mu)\left(\sum_{k \neq i, j}\left[\left(x_{k}-x_{i}\right)^{2}-\left(x_{k}-x_{j}\right)^{2}\right]+0-\left(x_{i}-x_{j}\right)^{2}\right) \\
& +\frac{\mu}{2}\left(\sum_{k \neq i, j}\left[\left(x_{k}-x_{i}-1\right)^{2}-\left(x_{k}-x_{j}\right)^{2}\right]+1-\left(x_{i}-x_{j}\right)^{2}\right) \\
& \left.+\frac{\mu}{2}\left(\sum_{k \neq i, j}\left[\left(x_{k}-x_{i}+1\right)^{2}-\left(x_{k}-x_{j}\right)^{2}\right]+1-\left(x_{i}-x_{j}\right)^{2}\right)\right]
\end{aligned}
$$

After some algebra, we get

$$
<\dot{\omega}>=\frac{2 N}{N+1}\left(-\frac{<\bar{x} \omega>}{N}+<C_{3}>+\mu<\bar{x}>\right)
$$

where $\bar{x}$ is the average $x$ value of the population, $(1 / N) \sum_{i} x_{i}$ and $C_{3}$ is the asymmetry, $(1 / N) \sum_{i}\left(x_{i}-\bar{x}\right)^{3}$. This asymmetry automatically vanishes in the case of 2 particles, so the equation reduces to the Equation (27) in that case. We have already seen that the average asymmetry vanishes at long times like $1 / t$, (an argument which easily extends to all orders in $\mu$ ), so that upon factorizing the expectation value $\langle\bar{x} \omega>$ as above, we get that the width asymptotically approaches $\mu N$.

We can understand simply the origin of each of the three terms in the equation of motion for $\langle\dot{\omega}\rangle$. The $\mu$ term comes from the fact that mutation acts to increase the width. Since only babies can mutate, the effective mutation rate is proportional to $\mu x$. The asymmetry term expresses the effect of selective pressure. If there are particles that lag behind, giving rise to a negative asymmetry, they are quickly killed off, decreasing the width. The last term, $\langle\bar{x} \omega>$, is more subtle. It expresses the fact that the population tends to collapse even in the absence of selective pressure. Death always acts to reduce population variability, whereas birth does not increase it. This effect is crucial for the stabilization of the width against the effects of mutation at long times. The rate associated with this effect is $2 \bar{x} /(N+1)$. As the velocity scales linearly in $N$, however, (which we have proved to lowest order in $\mu$ above and which we demonstrate below to all orders) the time scale for the asymptotic width dynamics is essentially independent of $N$. Unfortunately, this does not mean that the asymptotic state is necessarily approached in a time which is $N$ independent; we will see later that this issue is actually considerably more complex.

\section{B. The Drift Velocity}

It turns out that not only can we derive the width to all orders in $\mu$, we can also simply relate this width to the velocity of the average mean position, $\dot{\bar{x}}$.

The average mean position is given by

$$
<\bar{x}>=\frac{1}{N} \sum_{C} P_{C} \sum_{i} x_{i}
$$

Now a little reflection suffices to convince oneself that mutation does not contribute to the instantaneous velocity, since the probabilities for mutating left and right are identical. Thus the entire velocity is due to the collapse events. Then, as above, summing over all events where $i$ has a baby, resulting in the death of $j$,

$$
<\dot{\bar{x}}>=\frac{1}{N(N+1)} \sum_{C} \sum_{i} \sum_{j} P_{C} x_{i}\left(x_{i}-x_{j}\right)
$$




$$
\begin{aligned}
& =\frac{1}{N(N+1)} \sum_{C} \sum_{i} \sum_{j} P_{C}\left(x_{i}^{2}-x_{i} x_{j}\right) \\
& =\frac{N-1}{2(N+1)}\langle\omega\rangle
\end{aligned}
$$

Thus, the velocity is just exact proportional to $\langle\omega\rangle$, the average squared-width! This is physically reasonable: the velocity arises from the collapse of the population onto the more fit members. The wider the distribution, the faster the average fitness increases. Now, we found above the asymptotic value of $\langle\omega\rangle$ to be $\mu N$, yielding the striking result that the velocity is exactly linear in $\mu$, and given by our lowest order result, Eq. (17) found above!

Turning back to the simulation results in Fig. 2, we see that the results are indeed consistent with a velocity converging to the theoretical value, Eq. (17). Extending the run to longer times confirms this result. Similarly, we see the squared-width tracks with the velocity as predicted, increasing toward the expected value $\mu N$.

We can also calculate in a similar manner the diffusion constant $D$ of the center of mass motion. From Eq. (16) above, one can deduce that $\left.d / d t\left(<(\bar{x})^{2}>-<\bar{x}\right\rangle^{2}\right)=2 D<\bar{x}>$. Calculating this quantity directly via the moments, we find

$$
\frac{d}{d t}\left(<(\bar{x})^{2}>-<\bar{x}>^{2}\right)=\frac{1}{N+1}\left(<C_{3}>+\frac{N-1}{N}<\bar{x} \omega>+\mu<\bar{x}>\right)
$$

Since, at long times, $\left\langle C_{3}\right\rangle$ decays to zero, and $\langle\omega\rangle$ approaches $\mu N$, we get that

$$
D=\frac{\mu N}{2(N+1)}
$$

again reproducing our lowest order result exactly.

So, we have been able to show that the asymptotic state of the evolution process is one in which the width saturates to a fixed value, leading to a pulse propagating at constant velocity in fitness space. The exact values of the velocity and width have been determined. What we still need to discuss is the failure of mean-field theory and the related issue of the transient behavior before the asymptotic state sets in. We will return to these issues shortly. Now, we briefly detour to comment on the flat landscape case.

\section{THE FLAT LANDSCAPE AND OTHER VARIANTS}

All the analysis above works as well (and more simply) in the case of the flat landscape; i.e., where the birth rate is independent of $x$. A variant of this flat-landscape model in which not only babies mutate, but every individual does, was considered first by Zhang, et. al [9] and exactly solved by Meyer, Havlin, and Bunde [10]. In this variant, the relevant parameter is the ratio of the mutation rate to the birth rate, which we may take to be unity. This ratio we label $\tilde{\mu}$, to distinguish it from $\mu$, the probability that a baby mutates. The context in which this variant was analyzed was a problem of population migration, so that the "mutation" occurs in physical space, not "fitness" space. One can consider also an intermediate case, where everyone mutates (or migrates) and the birth rate depends on position, (the "emigration" problem, where people look for "greener" pastures). All these variants have many features in common. As we shall see, the major differences between the models are 1)the nature of the "drift", or velocity; and 2)the time-scale for the wave-function to reach its asymptotic width.

The flat landscape model of Zhang, et. al. and Meyer, et. al. is sufficiently simple to allow for a complete analytic solution, which Meyer et. al. present in a lovely piece of work 11. One essential difference in the flat landscape case is that there is no drift. The equation of motion for the center of mass in the long-time limit is exactly the diffusion equation:

$$
\dot{f}=D f^{\prime \prime}
$$

where the diffusion constant $D=\tilde{\mu} / 2$ is exactly that of a single individual. The explanation for this fact, already noted by Zhang, et. al., is that the collapse event leaves the parent unaffected, so that there is at long times one "ur-individual" who just propagates as if nothing ever happened. The localization of the wavefunction then implies that all the diffusion of the center of the mass is due just to the diffusion of this "ur-individual". Comparing this to our result we see that this parallels our results for the diffusion constant in the "tilted" case. There we found that the diffusion constant was $N \mu x / 2(N+1)$. This is just the effective diffusion constant for a single individual, since in 
the babies-only model, a diffusion step only occurs if the baby is not immediately killed, so that the effective rate of mutation is $\mu N /(N+1)$ times the birth rate $x$, giving rise to a diffusion constant as noted above. The fact that only babies mutate is not an essential change since the dynamics would be the same if only the parent mutated, so again only the motion of the "ur-individual" need be considered.
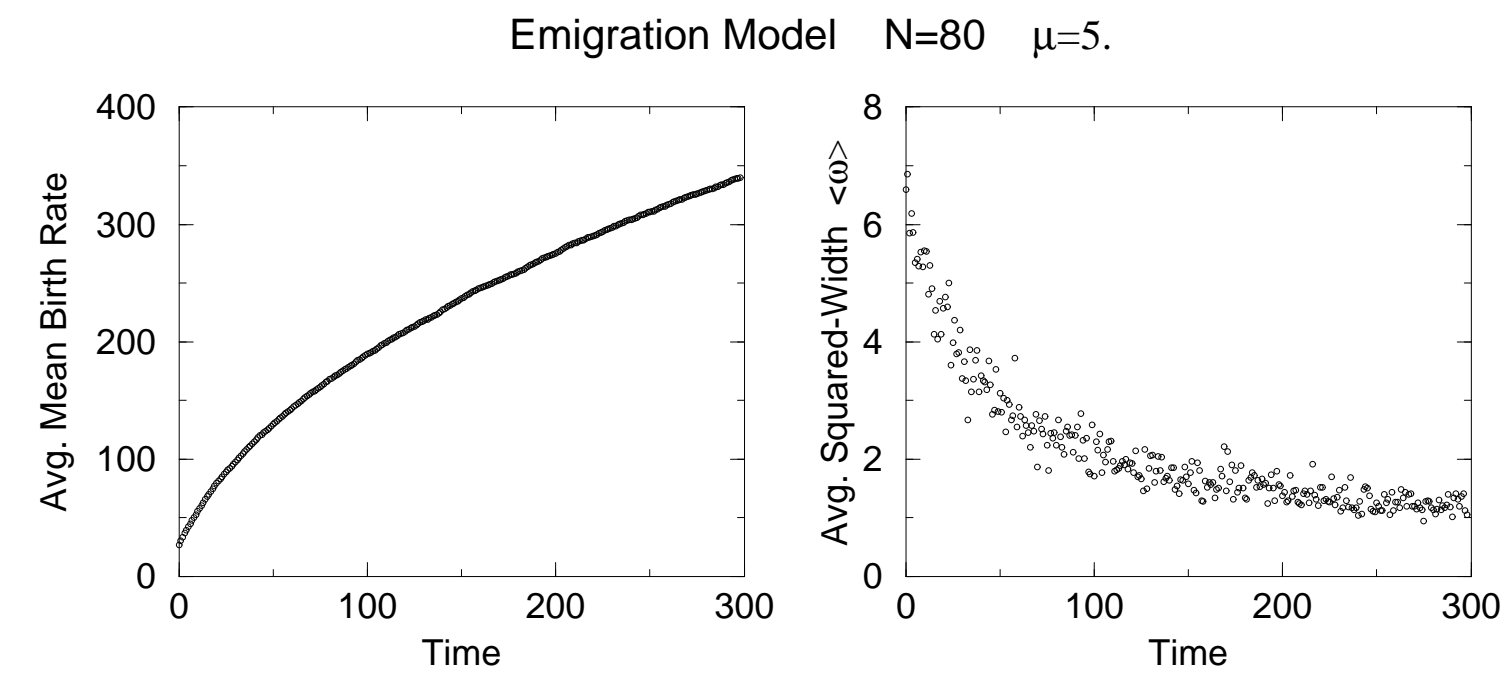

FIG. 3. a) Mean birth-rate for population of $N=80$ individuals as a function of time in the "emigration" model, with mutation rate $\mu=5$., averaged over 100 realizations.

b) Width-squared from same simulation.

Of course, the drift must vanish in the flat-landscape case, by virtue of the left-right symmetry. In the intermediate model where all individuals can mutate, but the landscape is tilted, it is straightforward to see that the velocity is proportional to $1 / \bar{x}$, the current average position, so that the position should scale as $\sqrt{t}$. This is related to the fact that the width of the wave function decreases as $1 / \bar{x}$. The point is that a constant mutation rate is not effective enough to combat the ever-increasing suppression of variability due to the ever-increasing birth/death rate. This behavior is apparent in the simulation data of this model for $N=80$ presented in Fig. 3. We will return to a discussion of this "emigration" model in Sec. VII.

As already mentioned, the third order moment $C_{3}$ in the width equation represents the effect of selective pressure. Is is therefore absent in the flat landscape case; instead, the moment equation for the width squared, $\omega$, reads

$$
<\dot{\omega}>=2\left(-\frac{<\omega>}{N}+\tilde{\mu}\right)
$$

Also missing, naturally, are the factors of $\bar{x}$. Thus the moment equation closes, allowing for an exact solution, identical to that found by Meyer, et. al.

The last major difference worth noting is the time scale for the asymptotic width dynamics. We saw how in the tilted case the time scale is $\bar{x} / N$. The factor $\bar{x}$ is the effective birth rate. In the flat landscape case, the birth rate is constant, so the time scale is fixed and or order $1 / N$. Thus stabilization of the width in the flat landscape case always takes place after $O(N)$ time.

\section{MOMENT EQUATIONS AND MEAN-FIELD THEORY}

The equations of motion we have derived for the width and velocity not only allow us to calculate the asymptotic behavior of these quantities. They also allow us to make contact with the mean-field theory we presented in section IIB. The point is that one can derive parallel equations for these moments directly from the mean-field (MF) theory. By comparing the exact equations with the approximate ones obtained from the mean-field theory, we can obtain some insight into the breakdown of the mean-field theory, and how to improve upon it. 
The MF equation for the velocity is easy to obtain. The mean of $x$ is

$$
<x>_{\mathrm{MF}}=\sum n P(n)
$$

Using the MF equation of motion for $P$, Eq. (2), we obtain

$$
<\dot{x}>_{\mathrm{MF}}=C_{2}^{\mathrm{MF}}
$$

where $C_{2}^{\mathrm{M} F}$ is the second cumulant, $<n^{2}>_{\mathrm{M} F}-<n>_{\mathrm{M} F}^{2}$. This second cumulant is, to leading order in $1 / N$, just $\omega / 2$. Thus, comparing with the exact equation, Eq. (33), we see that the MF result is exact up to trivial $1 / N$ corrections.

We can obtain in a similar manner an equation of motion for the second cumulant. We find

$$
\dot{C}_{2}^{\mathrm{M} F}=C_{3}^{\mathrm{M} F}+\mu<x>_{\mathrm{M} F}
$$

Comparing with the exact equation for $\omega$, Eq. (31) we see the equations correspond except for the absence of the stabilizing term $-\left\langle x C_{2}>/ N\right.$. Now of course this term is formally of order $1 / N$, and so the MF cannot be expected to reproduce it [12]. Nevertheless, we have seen that this is the term responsible for the saturation of the width at long times. The point is that $\left\langle C_{2}\right\rangle$ is $O(N)$ at long times and so the term is in truth not negligible.

The question at this point is, how long is long? When does this and other formally small terms become relevant? The answer is very quickly, on a time scale of $O(1)$. The reason for this is the blowup of MF we alluded to in section 2. We can see this blowup directly by solving exactly the MF equation of motion, Eq. (2). To do this, we define a generating function for the moments, $Z(a, t)$,

$$
Z(a, t) \equiv \sum_{n} P(n, t) e^{a n}
$$

It is actually more convenient to work in terms of the function $F \equiv \ln Z$, which is a generating function for the cumulants. Plugging the definition of $F$ in the equation of motion for $P$, we find

$$
\dot{F}(a, t)=(1+\mu \cosh (a)-\mu) \frac{d}{d a} F(a, t)-\left.\frac{d F}{d a}\right|_{a=0}
$$

We can solve this equation by means of defining a new variable $s$ such that

$$
\frac{d s}{d a}=\frac{1}{1+\mu \cosh (a)-\mu}
$$

so that

$$
s(a)=\frac{1}{\sqrt{1-2 \mu}} \ln \left(\frac{1+\sqrt{1-2 \mu} \tanh (a / 2)}{1-\sqrt{1-2 \mu} \tanh (a / 2)}\right)
$$

for $\mu<1 / 2$ and

$$
s(a)=\frac{2}{\sqrt{2 \mu-1}} \arctan (\sqrt{2 \mu-1} \tanh (a / 2))
$$

for $\mu>1 / 2$. In terms of $\mathrm{s}$, the equation for $F$ reads

$$
\dot{F}(s, t)=\frac{d}{d s} F(s, t)-\left.\frac{d F}{d s}\right|_{s=0}
$$

whose solution is

$$
F(s, t)=F_{0}(s+t)-F_{0}(t)
$$

where $F_{0}(s) \equiv F_{0}(a(s))$ is the generating function for the cumulants of $P(n, t=0)$. For example, if we start with a zero width pulse located at $n_{0}$ at time $t=0, F_{0}(s)=n_{0} a(s)$. Then, translating back to our original variable $a$, we find (for our zero width pulse) 


$$
F(a, t)=n_{0}\left(s^{-1}(s(a)+t)-s^{-1}(t)\right)
$$

The key feature of this solution is that $s_{\infty} \equiv s(a=\infty)$ is finite, so that the inverse function $s^{-1}$ is singular at $s_{\infty}$. This causes $F$ to diverge at time $t^{*}=s_{\infty}$. Explicitly,

$$
t^{*}= \begin{cases}\frac{1}{\sqrt{1-2 \mu}} \ln \left(\frac{1+\sqrt{1-2 \mu}}{1-\sqrt{1-2 \mu}}\right) & \mu<\frac{1}{2} \\ \frac{2}{\sqrt{2 \mu-1}} \arctan (\sqrt{2 \mu-1}) & \mu>\frac{1}{2}\end{cases}
$$

so that $t^{*}$ diverges as $-\ln \mu$ for $\mu \ll 1$ and decreases monotonically to $\pi / 2$ for the maximal $\mu=1$. For example, the first moment of $P$, the mean position, is given by

$$
<x>_{\mathrm{M} F}=\left.\frac{d F}{d a}\right|_{a=0}= \begin{cases}\frac{n_{0}}{\left(1-\frac{1}{\chi^{2}} \tanh ^{2}\left(\frac{\chi t}{2}\right)\right) \cosh ^{2}\left(\frac{\chi t}{2}\right)} & \mu<\frac{1}{2} \\ \frac{n_{0}}{\left(1-\frac{1}{\chi^{2}} \tan ^{2}\left(\frac{\chi t}{2}\right)\right) \cos ^{2}\left(\frac{\chi t}{2}\right)} & \mu>\frac{1}{2}\end{cases}
$$

where $\chi \equiv \sqrt{|1-2 \mu|}$. The divergence of this quantity at $t=t^{*}$ is apparent.

In fact, all nonzero cumulants of $P$ diverge at $t^{*}$, with the $j$ th cumulant diverging as $\left(t^{*}-t\right)^{-j}$. This makes an analysis of the precise manner of the breakdown of MF rather subtle. Consider the equation of motion for $\omega$, Eq. (31). It is true that $\left\langle\bar{x}>\omega / N\right.$ is diverging as $t \rightarrow t^{*}$ as $\left(t^{*}-t\right)^{-3}$, but then again the $C_{3}$ term in the equation is also diverging in exactly the same manner. As it is clear that MF must break down before $t=t^{*}$, it must be higher-order moments that are responsible. These higher-order moments are responsible for driving the low-order moments to diverge, and if they are cut off somehow by the $1 / N$ terms, the effect will eventually propagate down to the low-order moments.

It is difficult at this stage to pinpoint precisely the workings of this mechanism however. At issue is whether there exists an intermediate time regime at very large $N$ where neither MF nor the asymptotic regime with its constant velocity are accurate descriptions of the dynamics. To indicate the nature of the problem, we present in Fig. 4 a graph of the time-development of the squared-width, $\langle\omega\rangle$, as a function of time as measured from simulations for a number of different $N$ 's. The initial time regime with its MF-like behavior is clearly evidenced for short times. This rapid rise in the velocity is then abruptly cut off. The velocity then slowly increases to its asymptotic value. The time-scale of this slow rise, and its dependence on $N$ are not at this point clear to us. We hope to return to these issues in a future work.

\section{THE “EMIGRATION" MODEL}

We now examine the breakdown of MF for the variant model where everyone mutates, the "emigration" model. As we indicated in Section VI, the asymptotic state is characterized by a velocity decreasing as $1 / \sqrt{t}$. The short-time dynamics is controlled by the following MF equation:

$$
\dot{P}(n)=(n-\bar{n}) P(n)+\frac{1}{2} \tilde{\mu}[P(n+1)-2 P(n)+P(n-1)]
$$

We can also solve this MF exactly. The equation for $F(a)$ now reads

$$
\dot{F}(a)=\frac{d}{d a} F(a)+\tilde{\mu}(\cosh (a)-1)-\left.\frac{d F}{d a}\right|_{a=0}
$$

For an initial population located at $n_{0}$, so that $F(a, t=0)=n_{0} a$, one can verify that

$$
F(a, t)=\tilde{\mu}(\sinh (a+t)-\sinh (t)-\sinh (a))+n_{0} a
$$

satisfies the MF equation. From this solution, one can read off that the velocity $v=\tilde{\mu} \sinh (t)$ grows exponentially in time. Thus, for this model, MF must break down by the time $\ln (N / \tilde{\mu})$ since at this time the MF value of $\langle x\rangle\langle\omega\rangle / N$ is competitive with the $C_{3}$ term as given by MF. However, as with the only-babies-mutate model analyzed in the previous section, the breakdown of MF appears to be more complex. Simulations indicate that in fact MF breaks down before this time and in particular when $\langle x\rangle\langle\omega\rangle / N$ is still small compared to the other terms. We present in Fig. 5, simulations of the width as a function of time for various $N$. Again, for very short times, we 
see a regime where MF is accurate. The growth of the width is then suddenly cut-off and the width starts decaying. The cutoff is seen to take place at times earlier than that indicated by the simple argument above, due to the role of higher-order moments, similar to what we found for the only-babies-mutate model as discussed above.

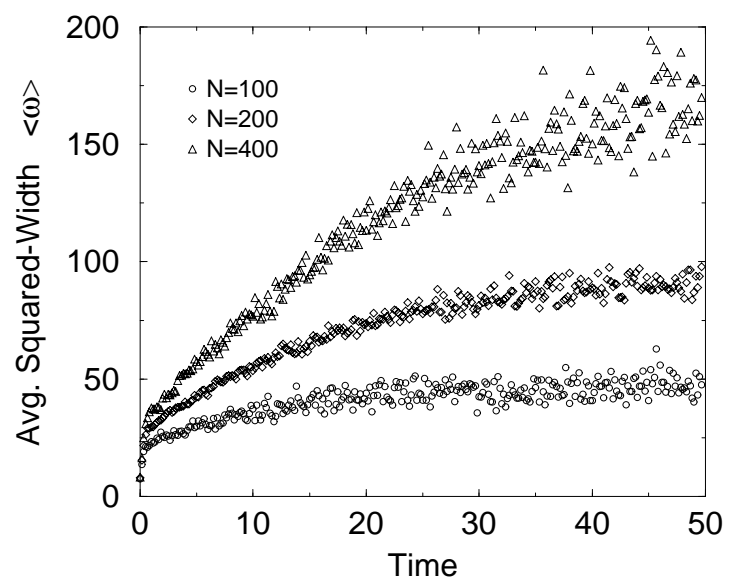

FIG. 4. Squared-width as a function of time for populations of $N=100$ (circle), $N=200$ (diamond), and $N=400$ (triangle) individuals, with mutation rate $\mu=.5$, averaged over 160,640 and 160 realizations respectively.

In our previous work [1], we presented an analysis of a cut-off version of the MF for this everyone-mutates model. The cut-off amounted to killing the $n-\bar{n}$ term for those sites where $P(n)$ was less than some threshold value [8]. This model possessed an asymptotic state which propagated at constant velocity. In fact, starting from a distribution localized on a single site $n_{0}$, the velocity in the cut-off theory increases monotonically to this asymptotic value. We see that this cutoff theory seriously misrepresents the actual physics, even at large $N$. In essence, this form of cutoff is too weak. We have seen that the corrections to MF are in fact stabilizing in nature, whereas the simple truncation of this cutoff just makes the growth at the leading edge marginal. The question of whether a more physically correct cutoff can be constructed is intimately related to the question of the precise nature of the breakdown of naive MF and the rapidity of approach to the asymptotic state; these questions we have chosen to postpone to future work.

\section{CONCLUSIONS}

The purpose of this paper was to present a detailed analysis of a simple model for evolution on a smooth landscape. This model was originally motivated by experiments on RNA viruses which showed persistent increases in fitness without the "punctuation" that should be present if the fitness landscape had significant roughness thereby giving rise to local maxima. Our results indicate that the system does settle into a asymptotic state characterized by a constant rate of fitness growth. This state is dominated by a balance between mutation and the the loss of variability within the population due to death. This latter effect, although formally weak in a large population, is crucial in rescuing the population from the runaway increase in birth-rate seen in the mean-field limit.

Our model exhibits certain generic features which should be sufficiently robust that it is reasonable to hope that they apply also to the biological system which motivated these investigations. The most striking is the role of the population size on the dynamics. Since the $N$-independent mean-field treatment breaks down so spectacularly after a very short time, we may expect that the population size is a very relevant parameter. The other prediction of our model that bears checking in the experimental system is the behavior of the population variance. We have seen that if the initial variance is large, there is a rapid initial collapse of variance. The variance then asymptotes to a fixed quantity and does not exhibit diffusional broadening in time. Also, the fact that the rate of improvement of fitness vanishes for small $N$ is very relevant for modelling the process of "genetic bottlenecking" [13] used to create the initial population in the experiment.

Our investigations have implications beyond the narrow confines of the model analyzed herein. The breakdown of mean-field theory exhibited by our model is shared by diffusion-limited aggregation. There also it is clear that this breakdown is the result of the neglect of correlations, in this case correlations between the walker density and the density of the aggregrate. This correlation is most apparent for particles who manage to outrun the typical extent of the aggregate. Whereas the ensemble-averaged walker density is quite large at the position of this outlying particle, 
the actual walker density is very small. The neglect of this correlation leads to a blowup of the mean-field exactly parallel to the blowup exhibited by the mean-field studied herein. The failure of simple cutoffs to correctly capture the asymptotic dynamics leads to the concern that similar cutoffs employed in the study of mean-field DLA might also misrepresent the dynamics of the system.

Our methods might also prove useful in the study of the behavior of genetic algorithms [14], where presents a similar system of evolving populations. Questions such as the speed of approach to the optimal state, the dependence on population size and the behavior of the population variance lie at the heart of understanding the working of these algorithms.

DAK thanks S. Havlin for sharing the results of his work prior to publication and I. Kanter for useful conversations. HL and DR acknowledge the support of the US NSF under grant DMR94-15460; LT was supported in part by DOE DE-FG03-95ER14516. DAK and HL acknowledge the support of the US-Israel Binational Science Foundation. DAK acknowledges the support of the Minerva Foundation.

[1] L. Tsimring, H. Levine, and D. A. Kessler, Phys. Rev. Lett. 76, 4440 (1996).

[2] I.S.Novella, E.A.Duarte, S.F.Elena, A.Moya, E.Domingo, and J.J.Holland, Proc. Natl. Acad. Sci. USA, 92, 5841-5844 (1995).

[3] M.Eigen and C.Biebricher, in: RNA Genetics, eds. E.Domingo, J.J.Holland, and P.Ahlquist (CPC Press, Boca Raton, FL, 1988).

[4] W. Fontana, W. Schnabl and P. Schuster, Phys. Rev. A 40, 3301 (1989).

[5] S.A.Kaufman, The Origins of Order, Oxford Univ.Press, New York, Oxford, 1993.

[6] T.A. Witten and L.M.Sander, Phys. Rev. B , 27, 5686 (1983).

[7] E.Brener, H.Levine, Y.Tu, Phys. Rev. Lett. , 66, 1978 (1991).

[8] A similar idea was put forth by T.B. Kepler and A.S.Perelson, Proc. Natl. Acad. Sci. USA, 92(18), 8219 (1995).

[9] Y.-C. Zhang, M. Serva, and M. Polikarpov, J. Stat. Phys. 58, 849 (1990).

[10] M. Meyer, S. Havlin, and A. Bunde, preprint, 1996.

[11] Meyer, et. al. only analyze the large $\tilde{\mu}$ limit, where the random walk of the individuals can be approximated by a diffusion equation. However, it is straightforward to generalize their analysis to the general $\tilde{\mu}$ case. The conclusions of such an analysis agree exactly with the results of the analysis in the text.

[12] It is possible to show that this $1 / N$ term does appear if one derives an equation coupling the mean field to the two-particle distribution function; this result will be presented elsewhere.

[13] H. J. Muller, Mut. Res. 1, 2 (1964).

[14] D. E. Goldberg, Genetic Algorithms in Search, Optimization, and Machine Learning, Addison-Wesley, Reading, MA., 1989. 